\title{
A New Era in the Quest for Dark Matter
}

\section{Gianfranco Bertone ${ }^{1}$ and Tim M.P. Tait ${ }^{1,2}$}

${ }^{1}$ GRAPPA Institute \& Institute of Physics, University of Amsterdam, Science Park 904, 1098 XH Amsterdam, The Netherlands

${ }^{2}$ Department of Physics and Astronomy, University of California, Irvine 92697, USA

\section{ABSTRACT}

There is a growing sense of 'crisis' in the dark matter community, due to the absence of evidence for the most popular candidates such as weakly interacting massive particles, axions, and sterile neutrinos, despite the enormous effort that has gone into searching for these particles. Here, we discuss what we have learned about the nature of dark matter from past experiments, and the implications for planned dark matter searches in the next decade. We argue that diversifying the experimental effort, incorporating astronomical surveys and gravitational wave observations, is our best hope to make progress on the dark matter problem.

\section{The Fall of Natural WIMPs}

The existence of dark matter has been discussed for more than a century ${ }^{1,2}$. In the 1970 s, astronomers and cosmologists have then began to build what is today a compelling body of evidence for this elusive component of the universe, based on a variety of observations that include temperature anisotropies of the Cosmic Microwave Background, baryonic acoustic oscillations, type Ia supernovae, gravitational lensing of galaxy clusters, and rotation curves of galaxies ${ }^{3,4}$. The Standard Model of particle physics contains no suitable particle to explain these observations, and thus dark matter arguably represents a glimpse of physics beyond the Standard Model (BSM). Proposed candidates for dark matter span 90 orders of magnitude in mass, ranging from ultra-light bosons, often referred to as "fuzzy dark matter" , to massive primordial black holes, a possibility that has received renewed interest after the LIGO and Virgo detection of gravitational waves from the merger of black holes several tens of times more massive than the Sun ${ }^{6,7}$.

The class of dark matter candidates that has attracted the most attention over the past four decades is weakly interacting massive particles (WIMPs). WIMPs appeared for a long time as a perfect dark matter candidate, as new particles at the weak scale would naturally be produced with the right relic abundance in the early universe ${ }^{8}$, while at the same time they might alleviate the infamous hierarchy problem ${ }^{9}$, that has been a main driver of particle physics for roughly four decades ${ }^{10}$. Despite much effort, no particle other than a Standard Model-like Higgs boson has been convincingly detected at the weak scale so far, a circumstance that, as long anticipated ${ }^{11}$, now raises the possibility that natural WIMPs may have been nothing more than an attractive red herring ${ }^{12}$.

The hierarchy problem is a consequence of the fact that quantum mechanics inevitably mixes up phenomena from all energy scales by allowing virtual particles to participate even in reactions whose energies are far too small to actually produce them. As a result, low energy quantities, such as the Higgs mass, can potentially receive very large corrections from the virtual influence of much heavier particles. The influence of heavy particles is particularly pronounced for scalar bosons such as the Higgs, and introduces corrections to the effective Higgs mass proportional to the masses of the virtual heavy states, such that the effective mass is the sum of a fundamental intrinsic value plus the correction terms. Since it is generally expected that new particles will appear at the Planck energy scale associated with 
quantum gravity, the observed Higgs mass at the weak scale appears highly unnatural, requiring an incredibly fine-tuned cancellation between the individually much larger intrinsic contribution and the correction terms, such that their sum is the value observed at the Large Hadron Collider. Natural theories introduce additional particles and symmetries which are arranged in such a way as to cancel off these large corrections amongst each other, protecting the Higgs mass from the influence of heavy mass scales.

The prototypical natural theory is the minimal supersymmetric (SUSY) standard model, which introduces an additional partner for each Standard Model particle. In addition, the partners of the electroweak bosons are predicted to be WIMPs, and thus are natural dark matter candidates. However, most of the parameter space of natural simple supersymmetric models is essentially ruled out ${ }^{13}$. Although it is still possible to identify 'natural' realizations of SUSY, e.g. in regions of parameter space of the phenomenological Minimal Supersymmetric Model $^{14}$, it is undeniable that null searches are constraining larger and larger portions of the parameter space of supersymmetric theories, which begs the question of how much fine-tuning one is willing to accept before giving up the hope to discover SUSY. ${ }^{15}$

\section{Alternatives to Natural WIMPs}

Non-natural WIMPs As a result of the lack of evidence for supersymmetry, naturalness is beginning to lose its luster as the guiding principle to construct theories of physics beyond the Standard Model. While the shift in emphasis away from WIMPs arising from extensions of the SM that address naturalness is inevitable, WIMPs themselves remain viable dark matter candidates in an appropriate context. For example, there are types of interactions which lead to indirect and direct signals which are highly suppressed, though such particles remain accessible to the LHC provided their masses are sufficiently small $^{16}$. With naturalness removed as the primary guide to theories of WIMPs, they evolve into a more general class of particles that achieve the appropriate relic density through self-annihilations.

This wider definition of WIMP - a circumstance that is already reflected in the adoption of simplified models ${ }^{17}$ and effective field theories ${ }^{18}$ in the presentation of colliders results - leads to a richer landscape of phenomenology. For example, the range of WIMP masses expands to encompass masses as low as around $1 \mathrm{MeV}$, or as high as around $100 \mathrm{TeV}$. This wider parameter space demands new kinds of WIMP searches, such as scattering of WIMP-like particles with masses below $1 \mathrm{GeV}$ off of electrons ${ }^{19}$, or with superconductors $^{20}$, superfluids ${ }^{21}$, or Dirac materials ${ }^{22}$. These light dark matter particles would typically have already been observed if their annihilation cross sections directly into Standard Model particles were large enough to explain their abundance in the Universe. As a result, viable models typically invoke similarly light dark force carriers, into which the dark matter can annihilate, and which subsequently decay into Standard Model states. Since they have small masses and must interact at some level with the Standard Model particles, these dark force carriers can be probed by high intensity, low energy accelerators ${ }^{23}$. Another complementary avenue is the search for TeV energy gamma rays produced in the annihilation of ultra-heavy dark matter particles with the upcoming gamma-ray Cherenkov Telescope Array (CTA) ${ }^{24,25}$.

Axions Another very popular class of dark matter candidates is that of axions and axion-like candidates. Axions are light ultra-weakly coupled particles which arise as a byproduct in theories which solve the 'strong-CP problem.' The symmetries of the Standard Model of particle physics would allow for the strong nuclear force to include an electric dipole moment (edm) for the neutron, which would represent an asymmetry in the charge distributions of the quarks which make it up. However, measurements indicate that the neutron edm is about $10^{-10}$ times smaller than naively expected, begging for a dynamical explanation. The dynamics which would cancel the neutron edm also produce a new particle: the axion ${ }^{26}$.

Many constraints exist on axions and axion-like models. A class of searches typified by the ADMX experiment ${ }^{27}$ uses a magnetic field to convert the background of axions on the Earth into an electromagnetic 
signal, and has successfully excluded a window of axion parameter space with masses around $2 \mu \mathrm{eV}$, and future data taking is expected to probe masses up to about $40 \mu \mathrm{eV}$. In addition, there is vigorous theoretical activity exploring new ideas to probe a wider range of axion masses ${ }^{28-30}$.

Sterile Neutrinos Another well-motivated candidate is a sterile neutrino which experiences a diluted form of the weak nuclear force through mixing with the "ordinary" active neutrinos. Such particles are a typical ingredient in theories which explain the fact that neutrinos have been experimentally found to be massive, in contrast to the predictions of the Standard Model. While their residual weak interactions predict that they will ultimately decay, if their mass and mixing are both small enough, the decay may occur slowly enough that they remain in the Universe today as a form of dark matter. They can be produced in the early Universe through a variety of different physical mechanisms ${ }^{31-34}$ with an appropriate abundance.

While the lifetime of a sterile neutrino playing the role of dark matter must be long enough that the vast majority of such particles have not yet decayed, quantum mechanics dictates that some will decay more rapidly, leading to a source of mono-energetic photons with energy close to half of its mass. In fact, an unidentified emission line at $3.5 \mathrm{keV}$ in the stacked X-ray spectrum of 73 galaxy clusters has prompted the suggestion that it might be a hint of the decay of a sterile neutrino ${ }^{35}$, though debate about the origin of this line is still ongoing ${ }^{36}$. Future X-ray telescopes such as eRosita, XARM, Athena, and/or Lynx should help to clarify the origin of this emission ${ }^{37}$, and future accelerator searches such as SHIP will provide a complementary probe of the relevant parameter space.

No stone left unturned. There is a plethora of other possible explanations for the nature of dark matter (see Figure 1 for a diagrammatic representation), from fuzzy dark matter $\left(10^{-22} \mathrm{eV}\right)$ to gravitationally produced WIMPzillas $^{38}$, and from superfluid dark matter ${ }^{39}$ to macroscopic objects like Macros $\left(10^{22}-\right.$ $\left.10^{24} \mathrm{~g}\right)^{40}$ and primordial black holes $\left(10 M_{\odot}\right)$. In light of this situation, the new guiding principle should be "no stone left unturned": we should look for dark matter not only where theoretical prejudice dictates that we "must", but wherever we can. Casting a wider theoretical net offers the possibility to discover new classes of dark matter candidates and new experimental opportunities to search for them, and also helps assemble a "composite image" of everything we currently know about the space of possibilities consistent with measurements to date.

\section{Probing the Nature of Dark Matter with Astronomical Observations}

Departures from LCDM. Given the current absence of evidence for dark matter particles from laboratory experiments, it is of utmost importance to extract as much information as possible from astronomical observations. Dark matter couplings other than gravity with itself or with standard model particle, or a non-negligible velocity dispersion, could lead in principle to measurable differences between observations and LCDM predictions ${ }^{41}$. It is in general important to search for "cracks" in the LCDM model, by carefully testing its underlying assumptions and observational predictions. An intriguing example is the discrepancy at the $3.7 \sigma$ level between cosmological ${ }^{3}$ and local measurements of the Hubble constant ${ }^{42}$.

We stress that great attention has to be paid not to mistake systematic errors in observations, or mismodelling of specific physical processes, for failures of the underlying LCDM model. It is perhaps not a surprise in this sense that most of the claimed problems of standard cosmology, such as the cuspcore, too-big-too-fail, and missing satellites problems ${ }^{41}$, arise in the deeply non-linear regime. Model predictions are in this case based on numerical simulations that encode complex processes like stellar formation, and supernova and black holes feedback, by means of an effective "sub-grid" description ${ }^{43}$, which is by construction a potential source of systematic errors. This shouldn't of course deter us from extensively testing the predictions of standard cosmology, by exploiting the wealth of information that 
will arise from upcoming astronomical surveys such as LSST, DESI, Euclid, and WFIRST, while at the same time improving the quality and predictive power of numerical simulations.

Self-interactions. A key property of dark matter that astronomical observations might help disproving is its collisionless nature. Dark matter self-interactions might actually help alleviate claimed tensions between numerical simulations and observations at small cosmological scales ${ }^{44,45}$. The imprint of dark matter self-interactions can be searched for in a number of ways. First, they can modify the shapes of dark matter halos ${ }^{44}$. Self-interactions tend in fact to make the central parts of dark matter halos more spherically symmetric than expected in collisionless scenarios. By comparing the shape of galaxy clusters in numerical simulations with that inferred from lensing and X-ray observations, it is possible to set an upper limit on the velocity-independent, elastic cross-sections of self-interacting dark matter $\sigma / m \gtrsim 1 \mathrm{~cm}^{2} \mathrm{~g}^{-146}$. Only very recently the first full simulations of galaxy clusters that incorporate both baryonic processes and dark matter self-interactions have been obtained ${ }^{47}$. Although much remains to be understood, it is encouraging that these simulations appear to support the analytical models tying the properties of self-interacting dark matter to the observed distribution of baryons ${ }^{48}$.

The trace of dark-matter self-interactions can also be searched for in merging systems such as cluster mergers and minor infalls ${ }^{49,50}$. The observables in this case would be the offset between the galaxies and the dark matter (in addition to the offset between dark matter and gas) due to the possible non-collisional nature of dark matter" ${ }^{1}$, and the amount of "sloshing" and "wobbling" of galaxies around the center of the dark matter halo ${ }^{41,52}$. As in the case of halo shapes, it is urgent to further investigate with full hydrodynamical simulations the complex interplay between gas cooling, AGN feedback, and dark matter physics, and understand the mapping between the properties of self-interacting dark matter and observables, in preparation of the wealth of observational data that will arise from upcoming astronomical surveys.

Substructures. A generic key property of dark matter in the standard cosmological model is that is is cold, i.e. non relativistic at the epoch of structure formation, and with a free streaming length much smaller than the size of galaxies. This implies the existence of a large number of sub-dwarf galaxy dark structures in galactic halos. If dark matter is warm, or more in general if its power spectrum is suppressed at small astrophysical scales, then we might discriminate it by probing the actual number of substructures in the Universe. A powerful probe of the power spectrum at small scales is Lyman-alpha forest in the spectra of high-redshift quasars ${ }^{53}$. This technique allows today to set a $2 \sigma$ lower limit on the warm dark matter particle mass of $5.3 \mathrm{keV}^{54}$, and on the mass of fuzzy dark matter particles of $37.5 \times 10^{-22} \mathrm{eV}^{55}$. New observations with the future high resolution spectrograph of the European Extremely Large Telescope (E-ELT), and with low resolution low signal-to-noise quasar spectra measured by DESI, should allow to substantially improve current bounds thanks to a larger statistical sample and a better determination of the thermal state of the intergalactic medium.

Another intriguing strategy to detect these dark substructures is the search for perturbations induced by sub-dwarf galaxy clumps on cold stellar streams ${ }^{56-58}$. Thanks to galaxy surveys like Gaia, currently taking data, and LSST, it should be in principle possible to detect impacts induced on stellar streams by subhalos with mass as low as $10^{7} M_{\odot}{ }^{59}$. By analyzing the power spectrum of the fluctuations of the stellar density in the stream future observations might even allow us to probe subhalos down to $10^{5} M_{\odot}{ }^{58}$. This method should allow to set stringent constraints on the mass of thermal dark matter relics with LSST data, and possibly yield an actual measurement of the dark matter particle mass if it is in the $\mathscr{O}(1) \mathrm{keV}$ range $\mathrm{e}^{60}$.

A more direct way of detecting dark matter substructures is via gravitational lensing. Although dark matter subhalos are not compact enough to be detectable e.g. with microlensing searches, they can modify the flux ratio of multiply lensed quasars ${ }^{61-64}$, and are potentially detectable via gravitational imaging, as a perturbation of magnified arcs and Einstein rings ${ }^{65}$. On top of lens substructures, low-mass dark matter 
halos along the line-of-sight can act as perturbers, and dominate the signal by an amount that depends on the lensing configuration and the dark matter properties ${ }^{66}$. This field will soon be revolutionized by upcoming astronomical surveys. The LSST for instance is expected to detect more than 8000 lensed quasars, $13 \%$ of which would be quadruple lenses ${ }^{67}$, which should allow to probe the subhalo mass function below $10^{8} \mathrm{M}_{\odot}$, while observations in the optical/near infrared with Euclid and the E-ELT, as well as in the radio with ALMA and the global VLBI interferometers should allow to probe the subhalo mass function at high redhsift ${ }^{68}$.

\section{Gravitational Wave Portal}

Primordial Black Holes. The detection of gravitational waves ${ }^{69}$ has opened up new opportunities to explore the physics of dark matter ${ }^{70}$. First, it has been suggested that the binary black holes whose merger has produced the gravitational waves detected by LIGO might be primordial, i.e. they might have formed in the very early universe, before Big Bang Nucleosynthesis $6,7,71$. The rate of binary black hole mergers would however be too high if such primordial black holes made up all of the dark matter in the Universe $^{72-74}$, a possibility that is also disfavored from a variety of constraints ranging the dynamical heating of dwarf galaxies, to distortions of the Cosmic Microwave Background, and from Supernova lensing to radio and X-ray emission due to the accretion of interstellar gas onto PBHs ${ }^{75}$. Although the constraints are becoming stringent, it is important to search for these objects even if they represent a subdominant component of dark matter. For instance, if we could discover a population of these objects in the universe, we would know that dark matter is not made of WIMPs, otherwise we should have already detected the annihilation radiation produce by WIMPs around them ${ }^{76}$. A number of observations, like the identification of BHs lighter than 1 solar mass, or the existence of BHs at redshift $z \gtrsim 40^{77}$, may in principle provide strong evidence for the existence of PBHs.

Constraints on modified gravity. Since the pioneering work on MOdified Newtonian Dynamics (MOND) in $1982^{78}$, numerous attempts have been made (e.g. Modified Gravity (MOG) ${ }^{79}$, and Emergent Gravity $^{80}$ ) to get rid of dark matter by modifying Einstein's theory of General Relativity. The success of these efforts however remained limited at most to rotation curves of galaxies, and it is today clear that the only way these theories can be reconciled with observations is by effectively, and very precisely, mimicking the behavior of cold dark matter on cosmological scales. The coincident observation of gravitational waves and electromagnetic radiation from GW $170817^{81}$ has allowed to set very stringent constraints on the propagation velocity of gravitational waves. The fact that it does not differ from the speed of light by more than one part in $10^{15}$ severely constrains all theories of modified gravity in which gravitational waves travel on different geodesics with respect to photons and neutrinos ${ }^{82-84}$. This has in particular allowed to rule out Bekenstein's Tensor-Vector-Scalar (TeVeS) theory ${ }^{85}$.

BH environment. Interestingly, dark matter might manifest itself as a perturbation in the waveform of binary black holes. If dark matter is made of cold and collisionless particles then their density around black holes will inevitably be higher than on average in the universe, and possibly much higher. Supermassive black holes at the center of galaxies might in particular host dark matter "spikes" 86 , although dynamical effects such as mergers with other black holes and interactions with stellar cusps might disrupt them ${ }^{87,88}$. Large dark matter overdensities are possible around Intermediate mass black holes ${ }^{89}$, and around primordial black holes ${ }^{90}$. The presence of dark matter around BHs would modify the dynamics of the merger, and induce a potentially detectable dephasing in the waveform ${ }^{70}$.

If dark matter is made of ultralight bosons, as in the aforementioned case of fuzzy dark matter, the field "cloud" that forms around black holes with a mass comparable to the Compton wavelength of 
bosons can be revealed in the gravitational waves signal from single or binary black holes, through direct monochromatic emission, stochastic background or through gaps in the black hole mass-spin Regge plane $^{91-93}$. Future analyses will allow to further elucidate possible "environmental" effects due to dark matter particles, and to discriminate among different dark matter models ${ }^{70}$.

\section{The Future}

In the quest for dark matter, naturalness has been the guiding principle since the dark matter problem was established in the early 1980s. Although the absence of evidence for new physics at the LHC does not rule out completely natural theories, we have argued that a new era in the search for dark matter has begun, the new guiding principle being "no stone left unturned": from fuzzy dark matter $\left(10^{-22} \mathrm{eV}\right)$ to primordial black holes $\left(10 M_{\odot}\right)$, we should look for dark matter wherever we can. It is important to exploit to their fullest extent existing experimental facilities, most notably the LHC, whose data might still contain some surprises. And it is important to complete the search for WIMPs with direct detection experiments, until their sensitivity reaches the so-called neutrino floor ${ }^{94}$.

At the same time we believe it is essential to diversify the experimental effort, and to test the properties of dark matter with gravitational waves interferometers and upcoming astronomical surveys, as they can provide complementary information about the nature of dark matter. New opportunities in extracting such information from data arise from the booming field of machine learning, which is currently transforming many aspects of science and society. Machine learning methods have been already applied to a variety of dark matter-related problems, ranging from the identification of WIMPs from particle and astroparticle data $^{95,96}$ to the detection of gravitational lenses ${ }^{97}$, and from radiation patterns inside jets of quarks and gluons at the $\mathrm{LHC}^{98}$ to real-time gravitational waves detection ${ }^{99}$. In view of this shift of the field of dark matter searches towards a more data-driven approach, we believe it is urgent to fully embrace, and whenever possible to further develop, big data tools that allow to organize in a coherent and systematic way the avalanche of data that will become available in particle physics and astronomy in the next decade.

\section{Acknowledgements}

We thank Vitor Cardoso, Daniele Gaggero, David Harvey, Dan Hooper, Bradley Kavanagh, Simona Vegetti, and Matteo Viel for useful comments on the initial version of this manuscript. The work of TMPT is supported in part by NSF grant PHY-1316792.

\section{References}

1. Bertone, G. \& Hooper, D. A History of Dark Matter. Submitt. to: Rev. Mod. Phys. (2016). 1605. 04909.

2. de Swart, J. G., Bertone, G. \& van Dongen, J. How dark matter came to matter. Nat. Astron. 1, 0059 (2017). DOI 10.1038/s41550-017-0059. 1703.00013.

3. Ade, P. A. R. et al. Planck 2015 results. XIII. Cosmological parameters. Astron. Astrophys. 594, A13 (2016). DOI 10.1051/0004-6361/201525830. 1502.01589.

4. Bertone, G. et al. Particle Dark Matter: Observations, Models and Searches (Cambridge Univ. Press, Cambridge, 2010). URL http://www. cambridge.org/uk/catalogue/catalogue. asp?isbn=9780521763684.

5. Hui, L., Ostriker, J. P., Tremaine, S. \& Witten, E. Ultralight scalars as cosmological dark matter. Phys. Rev. D95, 043541 (2017). DOI 10.1103/PhysRevD.95.043541. 1610.08297. 
6. Bird, S. et al. Did LIGO detect dark matter? Phys. Rev. Lett. 116, 201301 (2016). DOI 10.1103/PhysRevLett.116.201301. 1603.00464.

7. Clesse, S. \& García-Bellido, J. Detecting the gravitational wave background from primordial black hole dark matter. Phys. Dark Universe 18, 105-114 (2017). DOI 10.1016/j.dark.2017.10.001. 1610.08479.

8. Bertone, G., Hooper, D. \& Silk, J. Particle dark matter: Evidence, candidates and constraints. Phys. Rept. 405, 279-390 (2005). DOI 10.1016/j.physrep.2004.08.031. hep-ph / 0404175.

9. de Gouvea, A., Hernandez, D. \& Tait, T. M. P. Criteria for Natural Hierarchies. Phys. Rev. D89, 115005 (2014). DOI 10.1103/PhysRevD.89.115005. 1402 . 2658.

10. Dine, M. Naturalness Under Stress. Ann. Rev. Nucl. Part. Sci. 65, 43-62 (2015). DOI 10.1146/annurevnucl-102014-022053. 1501.01035.

11. Bertone, G. The moment of truth for WIMP Dark Matter. Nat. 468, 389-393 (2010). DOI 10.1038/nature09509. 1011.3532.

12. Giudice, G. F. The Dawn of the Post-Naturalness Era (2017). URL http: / / inspirehep . net / record/1631976/files/arXiv:1710.07663.pdf.1710.07663.

13. Athron, P. et al. Global fits of GUT-scale SUSY models with GAMBIT. Eur. Phys. J. C77, 824 (2017). DOI 10.1140/epjc/s10052-017-5167-0. 1705.07935.

14. van Beekveld, M., Beenakker, W., Caron, S., Peeters, R. \& Ruiz de Austri, R. Supersymmetry with Dark Matter is still natural. Phys. Rev. D96, 035015 (2017). DOI 10.1103/PhysRevD.96.035015. 1612.06333.

15. Ross, G. G., Schmidt-Hoberg, K. \& Staub, F. Revisiting fine-tuning in the MSSM. JHEP 03, 021 (2017). DOI 10.1007/JHEP03(2017)021. 1701.03480.

16. Goodman, J. et al. Constraints on Dark Matter from Colliders. Phys. Rev. D82, 116010 (2010). DOI 10.1103/PhysRevD.82.116010. 1008.1783.

17. Abdallah, J. et al. Simplified Models for Dark Matter Searches at the LHC. Phys. Dark Univ. 9-10, 8-23 (2015). DOI 10.1016/j.dark.2015.08.001. 1506.03116.

18. Beltran, M., Hooper, D., Kolb, E. W., Krusberg, Z. A. C. \& Tait, T. M. P. Maverick dark matter at colliders. JHEP 09, 037 (2010). DOI 10.1007/JHEP09(2010)037. 1002 . 4137.

19. Essig, R., Mardon, J. \& Volansky, T. Direct Detection of Sub-GeV Dark Matter. Phys. Rev. D85, 076007 (2012). DOI 10.1103/PhysRevD.85.076007. 1108.5383.

20. Hochberg, Y., Zhao, Y. \& Zurek, K. M. Superconducting Detectors for Superlight Dark Matter. Phys. Rev. Lett. 116, 011301 (2016). DOI 10.1103/PhysRevLett.116.011301. 1504.07237.

21. Knapen, S., Lin, T. \& Zurek, K. M. Light Dark Matter in Superfluid Helium: Detection with Multi-excitation Production. Phys. Rev. D95, 056019 (2017). DOI 10.1103/PhysRevD.95.056019. 1611.06228.

22. Hochberg, Y. et al. Detection of sub-MeV Dark Matter with Three-Dimensional Dirac Materials. Phys. Rev. D97, 015004 (2018). DOI 10.1103/PhysRevD.97.015004. 1708 . 08929.

23. Essig, R., Schuster, P. \& Toro, N. Probing Dark Forces and Light Hidden Sectors at Low-Energy e+eColliders. Phys. Rev. D80, 015003 (2009). DOI 10.1103/PhysRevD.80.015003. 0903.3941. 
24. Silverwood, H., Weniger, C., Scott, P. \& Bertone, G. A realistic assessment of the CTA sensitivity to dark matter annihilation. JCAP 1503, 055 (2015). DOI 10.1088/1475-7516/2015/03/055. 1408 . 4131.

25. Acharya, B. S. et al. Science with the Cherenkov Telescope Array. ArXiv e-prints (2017). 1709. 07997.

26. Abbott, L. F. \& Sikivie, P. A Cosmological Bound on the Invisible Axion. Phys. Lett. 120B, 133-136 (1983). DOI 10.1016/0370-2693(83)90638-X.

27. Du, N. et al. A Search for Invisible Axion Dark Matter with the Axion Dark Matter Experiment. Phys. Rev. Lett. 120, 151301 (2018). DOI 10.1103/PhysRevLett.120.151301. 1804 . 05750.

28. Kahn, Y., Safdi, B. R. \& Thaler, J. Broadband and Resonant Approaches to Axion Dark Matter Detection. Phys. Rev. Lett. 117, 141801 (2016). DOI 10.1103/PhysRevLett.117.141801. 1602. 01086.

29. Graham, P. W., Irastorza, I. G., Lamoreaux, S. K., Lindner, A. \& van Bibber, K. A. Experimental Searches for the Axion and Axion-Like Particles. Ann. Rev. Nucl. Part. Sci. 65, 485-514 (2015). DOI 10.1146/annurev-nucl-102014-022120. 1602.00039.

30. Caldwell, A. et al. Dielectric Haloscopes: A New Way to Detect Axion Dark Matter. Phys. Rev. Lett. 118, 091801 (2017). DOI 10.1103/PhysRevLett.118.091801. 1611.05865.

31. Shi, X.-D. \& Fuller, G. M. A New dark matter candidate: Nonthermal sterile neutrinos. Phys. Rev. Lett. 82, 2832-2835 (1999). DOI 10.1103/PhysRevLett.82.2832. astro-ph/9810076.

32. Laine, M. \& Shaposhnikov, M. Sterile neutrino dark matter as a consequence of nuMSM-induced lepton asymmetry. JCAP 0806, 031 (2008). DOI 10.1088/1475-7516/2008/06/031. 0804.4543.

33. Boyarsky, A., Ruchayskiy, O. \& Shaposhnikov, M. The Role of sterile neutrinos in cosmology and astrophysics. Ann. Rev. Nucl. Part. Sci. 59, 191-214 (2009). DOI 10.1146/annurev.nucl.010909.083654. 0901.0011.

34. Drewes, M. et al. A White Paper on keV Sterile Neutrino Dark Matter. JCAP 1701, 025 (2017). DOI 10.1088/1475-7516/2017/01/025. 1602.04816.

35. Bulbul, E. et al. Detection of An Unidentified Emission Line in the Stacked X-ray spectrum of Galaxy Clusters. Astrophys. J. 789, 13 (2014). DOI 10.1088/0004-637X/789/1/13. 1402 . 2301.

36. Jeltema, T. E. \& Profumo, S. Discovery of a $3.5 \mathrm{keV}$ line in the Galactic Centre and a critical look at the origin of the line across astronomical targets. Mon. Not. Roy. Astron. Soc. 450, 2143-2152 (2015). DOI 10.1093/mnras/stv768. 1408.1699.

37. Abazajian, K. N. Sterile neutrinos in cosmology. Phys. Rept. 711-712, 1-28 (2017). DOI 10.1016/j.physrep.2017.10.003. 1705.01837.

38. Kolb, E. W., Chung, D. J. H. \& Riotto, A. WIMPzillas! AIP Conf. Proc. 484, 91-105 (1999). DOI 10.1063/1.59655. [,592(1999)], hep-ph/9810361.

39. Berezhiani, L. \& Khoury, J. Theory of dark matter superfluidity. Phys. Rev. D92, 103510 (2015). DOI 10.1103/PhysRevD.92.103510. 1507.01019.

40. Kuhnel, F., Starkman, G. D., Freese, K. \& Matas, A. Primordial Black-Hole and Macroscopic Dark-Matter Constraints with LISA. ArXiv e-prints (2017). 1705.10361.

41. Buckley, M. R. \& Peter, A. H. G. Gravitational probes of dark matter physics. Submitt. (2017). 1712.06615. 
42. Riess, A. G. et al. New Parallaxes of Galactic Cepheids from Spatially Scanning the Hubble Space Telescope: Implications for the Hubble Constant. ArXiv e-prints (2018). 1801 . 01120.

43. Frenk, C. S. \& White, S. D. M. Dark matter and cosmic structure. Annalen der Physik 524, 507-534 (2012). DOI 10.1002/andp.201200212. 1210.0544.

44. Spergel, D. N. \& Steinhardt, P. J. Observational evidence for selfinteracting cold dark matter. Phys. Rev. Lett. 84, 3760-3763 (2000). DOI 10.1103/PhysRevLett.84.3760. astro-ph/ 9909386.

45. Tulin, S. \& Yu, H.-B. Dark Matter Self-interactions and Small Scale Structure. Phys. Rept. 730, 1-57 (2018). DOI 10.1016/j.physrep.2017.11.004. 1705.02358.

46. Brinckmann, T., Zavala, J., Rapetti, D., Hansen, S. H. \& Vogelsberger, M. The structure and assembly history of cluster-sized haloes in self-interacting dark matter. Mon. Not. Roy. Astron. Soc. 474, 746-759 (2018). DOI 10.1093/mnras/stx2782. 1705.00623.

47. Robertson, A. et al. The diverse density profiles of galaxy clusters with self-interacting dark matter plus baryons. Mon. Not. Roy. Astron. Soc. 476, L20 (2018). DOI 10.1093/mnrasl/sly024. 1711.09096.

48. Kaplinghat, M., Keeley, R. E., Linden, T. \& Yu, H.-B. Tying dark matter to baryons with selfinteractions. Phys. Rev. Lett. 113, 021302 (2014). URL https:// I ink.aps.org/doi/10. 1103 /PhysRevLett.113.021302. DOI 10.1103/PhysRevLett.113.021302.

49. Harvey, D., Massey, R., Kitching, T., Taylor, A. \& Tittley, E. The non-gravitational interactions of dark matter in colliding galaxy clusters. Sci. 347, 1462-1465 (2015). DOI 10.1126/science.1261381. 1503.07675 .

50. Robertson, A., Massey, R. \& Eke, V. Cosmic particle colliders: simulations of self-interacting dark matter with anisotropic scattering. Mon. Not. Roy. Astron. Soc. 467, 4719-4730 (2017). DOI 10.1093/mnras/stx463. 1612.03906.

51. Randall, S. W., Markevitch, M., Clowe, D., Gonzalez, A. H. \& Bradac, M. Constraints on the Self-Interaction Cross-Section of Dark Matter from Numerical Simulations of the Merging Galaxy Cluster 1E 0657-56. Astrophys. J. 679, 1173-1180 (2008). DOI 10.1086/587859. 0704.0261.

52. Harvey, D., Courbin, F., Kneib, J. P. \& McCarthy, I. G. A detection of wobbling Brightest Cluster Galaxies within massive galaxy clusters. Mon. Not. Roy. Astron. Soc. 472, 1972-1980 (2017). DOI 10.1093/mnras/stx2084. 1703.07365.

53. Narayanan, V. K., Spergel, D. N., Dave, R. \& Ma, C.-P. Constraints on the mass of warm dark matter particles and the shape of the linear power spectrum from the Ly $\alpha$ forest. Astrophys. J. 543, L103-L106 (2000). DOI 10.1086/317269. astro-ph/ 0005095.

54. Iršič, V. et al. New Constraints on the free-streaming of warm dark matter from intermediate and small scale Lyman- $\alpha$ forest data. Phys. Rev. D96, 023522 (2017). DOI 10.1103/PhysRevD.96.023522. 1702.01764 .

55. Iršič, V., Viel, M., Haehnelt, M. G., Bolton, J. S. \& Becker, G. D. First constraints on fuzzy dark matter from Lyman- $\alpha$ forest data and hydrodynamical simulations. Phys. Rev. Lett. 119, 031302 (2017). DOI 10.1103/PhysRevLett.119.031302. 1703.04683.

56. Yoon, J. H., Johnston, K. V. \& Hogg, D. W. Clumpy streams from clumpy halos: detecting missing satellites with cold stellar structures. The Astrophys. J. 731, 58 (2011). DOI 10.1088/0004$637 \mathrm{x} / 731 / 1 / 58$. 
57. Carlberg, R. G. Dark matter sub-halo counts via star stream crossings. The Astrophys. J. 748, 20 (2012). DOI 10.1088/0004-637x/748/1/20.

58. Bovy, J., Erkal, D. \& Sanders, J. L. Linear perturbation theory for tidal streams and the small-scale CDM power spectrum. Mon. Notices Royal Astron. Soc. 466, 628-668 (2017). DOI 10.1093/mnras/stw3067. 1606.03470.

59. Erkal, D. \& Belokurov, V. Properties of dark subhaloes from gaps in tidal streams. Mon. Notices Royal Astron. Soc. 454, 3542-3558 (2015). DOI 10.1093/mnras/stv2122.

60. Banik, N., Bertone, G., Bovy, J. \& Bozorgnia, N. Probing the nature of dark matter particles with stellar streams. ArXiv e-prints (2018). 1804.04384.

61. Mao, S. \& Schneider, P. Evidence for substructure in lens galaxies? Mon. Notices Royal Astron. Soc. 295, 587 (1998). DOI 10.1046/j.1365-8711.1998.01319.x. astro-ph/9707187.

62. Metcalf, R. B. \& Madau, P. Compound Gravitational Lensing as a Probe of Dark Matter Substructure within Galaxy Halos. The Astrophys. J. 563, 9-20 (2001). DOI 10.1086/323695. astro-ph/ 0108224 .

63. Dalal, N. \& Kochanek, C. S. Direct Detection of Cold Dark Matter Substructure. The Astrophys. J. 572, 25-33 (2002). DOI 10.1086/340303. astro-ph/0111456.

64. Gilman, D., Birrer, S., Treu, T. \& Keeton, C. R. Probing the nature of dark matter by forward modeling flux ratios in strong gravitational lenses. ArXiv e-prints (2017). 1712 . 04945.

65. Vegetti, S. \& Koopmans, L. V. E. Bayesian Strong Gravitational-Lens Modelling on Adaptive Grids: Objective Detection of Mass Substructure in Galaxies. Mon. Not. Roy. Astron. Soc. 392, 945 (2009). DOI 10.1111/j.1365-2966.2008.14005.x. 0805.0201.

66. Despali, G., Vegetti, S., White, S. D. M., Giocoli, C. \& van den Bosch, F. C. Modelling the line-ofsight contribution in substructure lensing. Mon. Not. Roy. Astron. Soc. 475, 5424-5442 (2018). DOI 10.1093/mnras/sty159. 1710.05029.

67. Oguri, M. \& Marshall, P. J. Gravitationally lensed quasars and supernovae in future wide-field optical imaging surveys. Mon.Not.Roy.Astron.Soc. 405, 2579-2593 (2010). DOI 10.1111/j.13652966.2010.16639.x. 1001.2037.

68. Daylan, T., Cyr-Racine, F.-Y., Diaz Rivero, A., Dvorkin, C. \& Finkbeiner, D. P. Probing the smallscale structure in strongly lensed systems via transdimensional inference. Astrophys. J. 854, 141 (2018). DOI 10.3847/1538-4357/aaaa1e. 1706.06111.

69. Abbott, B. P. et al. Observation of Gravitational Waves from a Binary Black Hole Merger. Phys. Rev. Lett. 116, 061102 (2016). DOI 10.1103/PhysRevLett.116.061102. 1602 . 03837.

70. Barack, L. et al. Black holes, gravitational waves and fundamental physics: a roadmap. ArXiv e-prints (2018). 1806.05195.

71. Carr, B., Kuhnel, F. \& Sandstad, M. Primordial Black Holes as Dark Matter. Phys. Rev. D94, 083504 (2016). DOI 10.1103/PhysRevD.94.083504. 1607.06077.

72. Sasaki, M., Suyama, T., Tanaka, T. \& Yokoyama, S. Primordial Black Hole Scenario for the Gravitational-Wave Event GW150914. Phys. Rev. Lett. 117, 061101 (2016). DOI 10.1103/PhysRevLett.117.061101. 1603.08338.

73. Ali-Haïmoud, Y., Kovetz, E. D. \& Kamionkowski, M. Merger rate of primordial black-hole binaries. Phys. Rev. D 96, 123523 (2017). DOI 10.1103/PhysRevD.96.123523. 1709.06576. 
74. Kavanagh, B. J., Gaggero, D. \& Bertone, G. Black Holes' Dark Dress: On the merger rate of a subdominant population of primordial black holes. ArXiv e-prints (2018). 1805.09034.

75. Gaggero, D. et al. Searching for Primordial Black Holes in the radio and X-ray sky. Phys. Rev. Lett. 118, 241101 (2017). DOI 10.1103/PhysRevLett.118.241101. 1612.00457.

76. Lacki, B. C. \& Beacom, J. F. Primordial Black Holes as Dark Matter: Almost All or Almost Nothing. Astrophys. J. 720, L67-L71 (2010). DOI 10.1088/2041-8205/720/1/L67. 1003.3466.

77. Koushiappas, S. M. \& Loeb, A. Maximum redshift of gravitational wave merger events. Phys. Rev. Lett. 119, 221104 (2017). DOI 10.1103/PhysRevLett.119.221104. 1708 . 07380.

78. Milgrom, M. A Modification of the Newtonian dynamics as a possible alternative to the hidden mass hypothesis. Astrophys. J. 270, 365-370 (1983). DOI 10.1086/161130.

79. Moffat, J. W. Scalar-tensor-vector gravity theory. JCAP 0603, 004 (2006). DOI 10.1088/14757516/2006/03/004. gr-qc/0506021.

80. Verlinde, E. P. Emergent Gravity and the Dark Universe. SciPost Phys. 2, 016 (2017). DOI 10.21468/SciPostPhys.2.3.016. 1611.02269.

81. Abbott, B. et al. GW170817: Observation of Gravitational Waves from a Binary Neutron Star Inspiral. Phys. Rev. Lett. 119, 161101 (2017). DOI 10.1103/PhysRevLett.119.161101. 1710.05832.

82. Boran, S., Desai, S., Kahya, E. O. \& Woodard, R. P. GW170817 Falsifies Dark Matter Emulators. Phys. Rev. D97, 041501 (2018). DOI 10.1103/PhysRevD.97.041501. 1710.06168.

83. Sakstein, J. \& Jain, B. Implications of the Neutron Star Merger GW170817 for Cosmological Scalar-Tensor Theories. Phys. Rev. Lett. 119, 251303 (2017). DOI 10.1103/PhysRevLett.119.251303. 1710.05893.

84. Wang, H. et al. The GW170817/GRB 170817A/AT 2017gfo Association: Some Implications for Physics and Astrophysics. Astrophys. J. 851, L18 (2017). DOI 10.3847/2041-8213/aa9e08. 1710.05805 .

85. Bekenstein, J. D. Relativistic gravitation theory for the MOND paradigm. Phys. Rev. D70, 083509 (2004). DOI 10.1103/PhysRevD.70.083509, 10.1103/PhysRevD.71.069901. [Erratum: Phys. Rev.D71,069901(2005)], astro-ph/ 0403694.

86. Gondolo, P. \& Silk, J. Dark matter annihilation at the galactic center. Phys. Rev. Lett. 83, 1719-1722 (1999). DOI 10.1103/PhysRevLett.83.1719. astro-ph/9906391.

87. Merritt, D., Milosavljevic, M., Verde, L. \& Jimenez, R. Dark matter spikes and annihilation radiation from the galactic center. Phys. Rev. Lett. 88, 191301 (2002). DOI 10.1103/PhysRevLett.88.191301. astro-ph/0201376.

88. Bertone, G. \& Merritt, D. Time-dependent models for dark matter at the Galactic Center. Phys. Rev. D72, 103502 (2005). DOI 10.1103/PhysRevD.72.103502. astro-ph/ 0501555.

89. Bertone, G., Zentner, A. R. \& Silk, J. A new signature of dark matter annihilations: gamma-rays from intermediate-mass black holes. Phys. Rev. D72, 103517 (2005). DOI 10.1103/PhysRevD.72.103517. astro-ph/0509565.

90. Ricotti, M., Ostriker, J. P. \& Mack, K. J. Effect of Primordial Black Holes on the Cosmic Microwave Background and Cosmological Parameter Estimates. Astrophys. J. 680, 829-845 (2008). DOI 10.1086/587831. 0709.0524. 
91. Brito, R. et al. Gravitational wave searches for ultralight bosons with LIGO and LISA. Phys. Rev. D96, 064050 (2017). DOI 10.1103/PhysRevD.96.064050. 1706.06311.

92. Arvanitaki, A., Baryakhtar, M., Dimopoulos, S., Dubovsky, S. \& Lasenby, R. Black Hole Mergers and the QCD Axion at Advanced LIGO. Phys. Rev. D95, 043001 (2017). DOI 10.1103/PhysRevD.95.043001. 1604.03958.

93. Baumann, D., Chia, H. S. \& Porto, R. A. Probing Ultralight Bosons with Binary Black Holes. ArXiv e-prints (2018). 1804.03208.

94. Billard, J., Strigari, L. \& Figueroa-Feliciano, E. Implication of neutrino backgrounds on the reach of next generation dark matter direct detection experiments. Phys. Rev. D89, 023524 (2014). DOI 10.1103/PhysRevD.89.023524. 1307.5458.

95. Bertone, G. et al. Identifying WIMP dark matter from particle and astroparticle data. JCAP 1803, 026 (2018). DOI 10.1088/1475-7516/2018/03/026. 1712.04793.

96. Caron, S., Kim, J. S., Rolbiecki, K., Ruiz de Austri, R. \& Stienen, B. The BSM-AI project: SUSYAI-generalizing LHC limits on supersymmetry with machine learning. Eur. Phys. J. C77, 257 (2017). DOI 10.1140/epjc/s10052-017-4814-9. 1605.02797.

97. Hezaveh, Y. D., Perreault Levasseur, L. \& Marshall, P. J. Fast Automated Analysis of Strong Gravitational Lenses with Convolutional Neural Networks. Nat. 548, 555-557 (2017). DOI 10.1038/nature23463. 1708.08842 .

98. Larkoski, A. J., Moult, I. \& Nachman, B. Jet Substructure at the Large Hadron Collider: A Review of Recent Advances in Theory and Machine Learning. ArXiv e-prints (2017). 1709.04464.

99. George, D. \& Huerta, E. A. Deep Learning for Real-time Gravitational Wave Detection and Parameter Estimation: Results with Advanced LIGO Data. Phys. Lett. B778, 64-70 (2018). DOI 10.1016/j.physletb.2017.12.053. 1711.03121. 


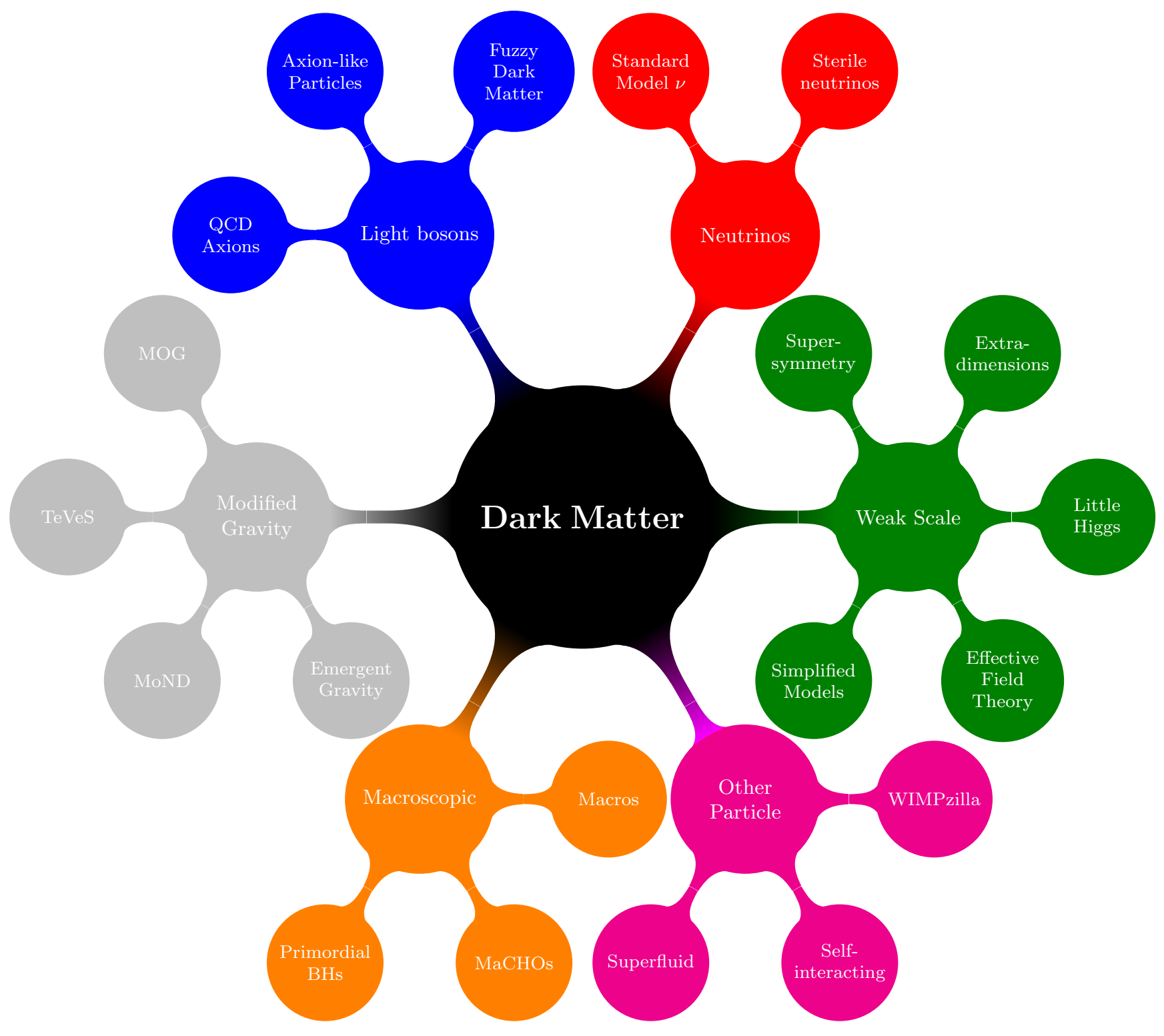

Figure 1. Visualization of possible solutions to the dark matter problem. 